\title{
Structural phase transitions and unusual melting behavior in a classical two-dimensional Coulomb bound cluster
}

\author{
W. P. Ferreira, ${ }^{1,2, *}$ B. Partoens ${ }^{2, \dagger}$ F. M. Peeters, ${ }^{2, \hbar}$ and G. A. Farias ${ }^{1, \S}$ \\ ${ }^{1}$ Departamento de Física, Universidade Federal do Ceará, Caixa Postal 6030, Campus do Pici, 60455-760 Fortaleza, Ceará, Brazil \\ ${ }^{2}$ Departement Fysica, Universiteit Antwerpen (Campus Drie Eiken), Universiteitsplein 1, B-2610 Antwerpen, Belgium
}

(Received 30 July 2004; published 9 February 2005)

\begin{abstract}
The melting properties of a cluster with $N$ equally charged particles confined by a Coulomb potential are studied. The system exhibits a structural phase transition before it melts. The melting process is not dominated by the usual thermal hops between stable states. We also show that the symmetry of the ground state configuration is a dominant factor in determining the melting temperature and that more confined particles in the cluster do not necessarily have a higher melting temperature.
\end{abstract}

DOI: 10.1103/PhysRevE.71.021501

PACS number(s): 83.10.Tv, 36.40.Ei, 64.90.+b

The melting phenomenon in three-dimensional (3D) crystals is significantly different from the melting in twodimensional (2D) crystals. In the latter case, and as pointed out by several authors, the transition from a solid phase to a complete isotropic liquid phase can be characterized by a two-step process with an intermediary hexatic phase [1]. However, such a melting scenario is not unique and depends on the systems. Furthermore, melting of 2D clusters is nonuniversal $[2,3]$.

For 2D finite crystals of charged particles, the melting also proceeds in a two-step process. Initially, there is an orientational disordering characterized by a relative rotation of neighboring shells, in which the internal order is retained. With further increasing of temperature, a radial melting is initiated, and a complete disordering in the system takes place (liquid phase). Such a behavior was observed in both a classical and quantum approach [4,5].

In general, the thermodynamic properties and the melting point of clusters are determined by a few low-lying metastable states (e.g., topological defects in strongly coupled two-dimensional systems $[1,6])$. Thermal fluctuations allow transitions between the ground state and these low-lying metastable states that are responsible for the breakdown of the ordered state. Such a scenario is applicable when the involved configurations are sufficiently robust to temperature fluctuations up to about the melting temperature. Furthermore, the height of the saddle points, separating the relevant configurations, should not be too large so that these thermal fluctuations can drive the system over these energy barriers.

In this paper, we investigate a system that does not follow the above melting scenario. The first characteristic of the system is that its ground state exhibits a very asymmetric configuration. Different metastable states may be present, but they are not essential for the complete melting process. A second important characteristic is the relative easy deformability of the configuration, i.e., it requires little energy to

\footnotetext{
*Electronic address: wandemberg@ fisica.ufc.br

${ }^{\dagger}$ Electronic address: bart.partoens@ua.ac.be

†Electronic address: francois.peeters@ua.ac.be

${ }^{\S}$ Electronic address: gil@ fisica.ufc.br
}

excite the normal modes. A very interesting feature of the present system is the dependence of the melting phenomenon on the symmetry of the ground state configuration. The key ingredient in our system to realize these properties, is a tunable confinement potential.

Our system consists of $N$ identically charged particles with charge $-e$, which are confined to move in a $2 \mathrm{D}$ plane and which are held together by a positive charge $Z e$ that is displaced a distance $d$ from the 2D plane. As compared to parabolically confined systems, this system has the advantage that it enables us to tune the strength of the confinement charge $Z$. In other words, we are considering a system in a nonuniform positive background, in contrast to the previous models that considered a uniform neutralizing positive background. The present system is not purely academic, but may be realized experimentally. For example, it is well known that electrons above a liquid helium surface form a 2D electron layer. Now one can insert impurities in the substrate supporting the helium film, which may confine the electrons laterally. $[7]$

The Hamiltonian of the system can be approximated by

$$
H=-\frac{Z e^{2}}{\epsilon} \sum_{i=1}^{N} \frac{1}{\sqrt{r_{i}^{2}+d^{2}}}+\frac{e^{2}}{\epsilon} \sum_{i>j=1}^{N} \frac{1}{\left|\mathbf{r}_{i}-\mathbf{r}_{j}\right|} .
$$

Here the symbol $\epsilon$ stands for the dielectric constant, and $\mathbf{r}$ $=\{x, y\}$ is the two component position vector of the 2D (twodimensional) particle. If we express the electron energy in units of $E_{0}=e^{2} / \epsilon d$ and all the distances in units of $d$, Eq. (1) can be rewritten in the following dimensionless form:

$$
H=-\sum_{i=1}^{N} \frac{Z}{\sqrt{r_{i}^{2}+1}}+\sum_{i>j=1}^{N} \frac{1}{\left|\mathbf{r}_{i}-\mathbf{r}_{j}\right|} .
$$

The temperature $T$ of the system will be considered in units of $T_{0}=E_{0} / k_{B}$, with $k_{B}$ Boltzmann's constant. The ground state configurations were found by using the Monte Carlo (MC) technique (Metropolis algorithm [8]) combined with a modified Newton method [9] to enhance the accuracy and the convergence. The particles were initially put in random positions within some circular area and then allowed to reach 
a steady state configuration after about $10^{5}$ simulation steps. To check if the obtained configuration is stable, we calculated the frequencies of the normal modes of the system [9]. The configuration was taken as final when all frequencies of the normal modes were real and the energy did not decrease further. In order to study melting, the system was heated up (where temperature was increased by steps of typically $\delta T$ $\approx 10^{-3}$ ) and equilibrated at the new temperature during 5 $\times 10^{4}-10^{5} \mathrm{MC}$ steps (one MC step corresponds with a displacement of all particles). After annealing, the average energy is calculated, together with the mean squared radial displacement

$$
\left\langle u_{R}^{2}\right\rangle \equiv \frac{1}{N} \sum_{i=1}^{N}\left(\left\langle r_{i}^{2}\right\rangle-\left\langle r_{i}\right\rangle^{2}\right) / a^{2},
$$

where $a$ is the average distance between the electrons at zero temperature. The symbol \langle\rangle stands for an average over typically $3 \times 10^{7} \mathrm{MC}$ steps after equilibration of the system. The quantity $\left\langle u_{R}^{2}\right\rangle$ gives us information about the average amplitude of the oscillating motion of the particles around their equilibrium positions. For a critical temperature, this quantity starts to increase in a fast manner, and when $\left\langle u_{R}^{2}\right\rangle \approx 0.1$ the system is considered melted, according to the modified Lindemann criterion [10].

Because of the nonparabolic nature of the confining potential, we showed earlier that this system exhibits very interesting properties as a function of $Z$, like structural phase transitions, overcharging, evaporation, and spontaneous symmetry breaking [11-13]. Nonsymmetric configurations were previously also found [14], but they were a result of a particular inter-particle interaction. The ground state, the metastable state, and the normal modes were studied previously and exhibited several interesting behavior, some of which did not have their equal in the well-studied parabolic confined dots $[4,9,15,16]$.

Here we consider temperature-induced phase transitions and melting in such clusters. An important question is: does the confinement strength determine the melting temperature, or is it the symmetry of the configuration that is more important? As will be shown, the melting can occur as a two-step process and the symmetry of the ground state configuration appears as an important factor determining the melting temperature $\left(T_{m}\right)$. In general, we found even structural phase transitions before the system melts completely.

In Fig. 1, the mean squared radial displacement is presented as a function of temperature for clusters with $N$ $=10,9,8,7,6,5$ particles and with constant positive confinement charge $Z=10$. The corresponding ground state configurations are presented in Fig. 2. Metastable states were not observed in these cases. Intuitively one expects that the neutral system $(N=Z=10)$, which has the smallest effective confinement energy per particle, has the lowest melting temperature $T_{m}$, while for the charged systems $(N=9,8,7,6,5)$ a higher $T_{m}$ is expected because they are stronger confined due to the net charge of the system. Surprisingly, such a behavior is not observed in Fig. 1 where it is shown that the cluster $N=9$ has a higher $T_{m}$ than the clusters $N=8$ and $N=7$, which have higher net charges. We found that the melting tempera-

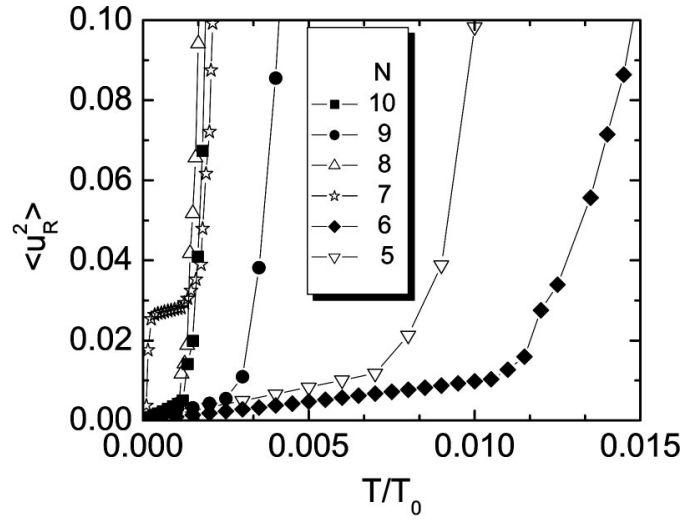

FIG. 1. The mean squared radial displacement as a function of temperature, considering clusters confined by a positive charge $Z$ $=10$, and with different number of particles.

ture is, to a large extent, determined by the symmetry of the ground state configuration and does not only depend on the confinement energy of the particles in the system, which we will discuss later. In order to characterize the symmetry of a configuration, the rotations that leave the configuration invariant are considered. A rotation operator over an angle $2 \pi / n$ is denoted by $C_{n}$, and the larger the value $n$ is for a configuration, the higher its symmetry. In the clusters $N$ $=10$ and $N=8$, which have the same configuration for the internal particles and are invariant under rotation $C_{2}$ [Figs. 2(a) and 2(c)], we observed practically the same melting temperature $T_{m} \approx 0.003$. The cluster $N=7$, which corresponds to an asymmetric arrangement of particles at $T=0$ (and thus only invariant under $C_{1}$ ), has a similar $T_{m}$. However, this cluster shows a qualitatively very different low-temperature behavior.

The cluster $N=9$, which is invariant under rotation $C_{3}$, has a larger $T_{m}$ than the less symmetric clusters $N=8$ (invariant under $C_{2}$ ) and $N=7$ (invariant under $C_{1}$ ). These facts made us conclude that the more packed and the more symmetric the particles are arranged, the larger the stability, and consequently, the larger the $T_{m}$. This is confirmed by investigating
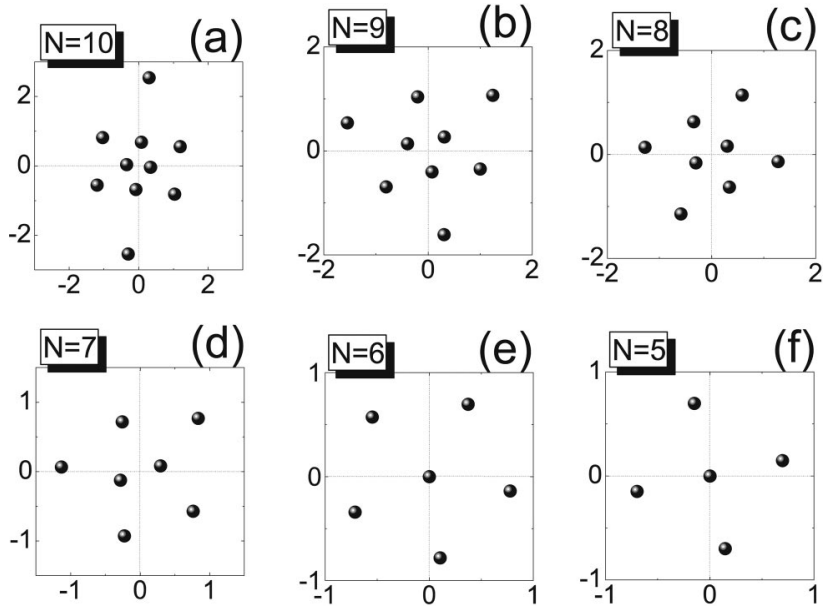

FIG. 2. Ground state configurations at $T=0$. All clusters are confined by a positive charge $Z=10$. 


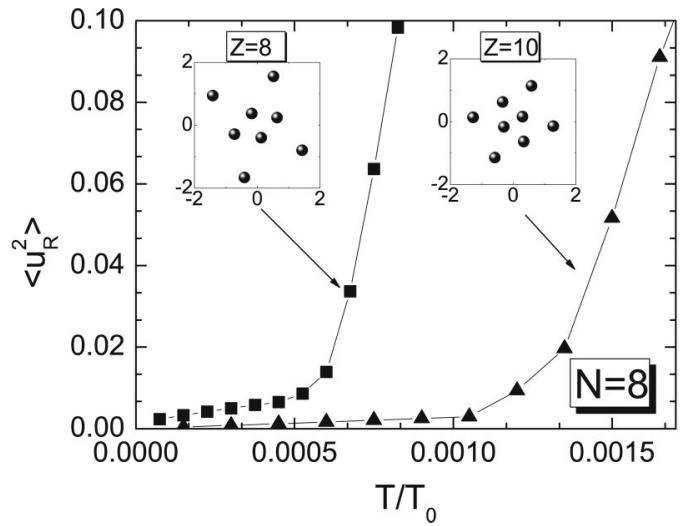

FIG. 3. The mean squared radial displacement as a function of temperature, for clusters consisting of $N=8$ particles, confined by positive charges $Z=8$ and $Z=10$. The ground state configurations at $T=0$ are shown as inset.

the cluster $N=6$, which has the largest value for $T_{m}$ (Fig. 1). Comparing the configurations of clusters $N=5$ (invariant un$\operatorname{der} C_{4}$ ) and $N=6$ (invariant under $C_{5}$ ), we observe that the last one is more symmetric and has the highest melting temperature. A similar behavior of the melting phenomenon was observed in other clusters with different values for $N$ and $Z$. But when the symmetry of the configurations are the same, the melting temperature is determined by the strength of the confinement potential, as shown in Fig. 3. The system $N=8$, $Z=10$ is more strongly confined than the system $N=Z=8$ and has a larger melting temperature. From the above results, we can conclude that the symmetry of the ground state configuration is the dominant factor in determining the melting temperature in small Coulomb confined clusters.

Now we will analyze more carefully the particular verylow-temperature region as observed for the cluster $N=7(Z$ $=10)$ in Fig. 1. Figure 4 shows, apart from the mean squared radial displacement [Fig. 4(a)], also the average radial position of each particle $\left\langle r_{i}\right\rangle$ [Fig. 4(b)], the mean squared radial displacement of each particle $\left\langle u_{R}^{2}\right\rangle_{i}$ [Fig. 4(c)] and the quantities $g_{6}$ [Fig. 4(d)] and $\left\langle u_{R}^{3}\right\rangle$ [Fig. 4(e)], which we will introduce later. Each particle is labeled by a number, as shown in the inset of Fig. 4(a). Note that at $T=0$, the ground state configuration consists of two shells, the inner ring forming a perfect circle, while the outer shell consists of three rings. For $T>0.0002 T_{0}$ a remarkable plateau in the mean squared radial displacement is observed (see Figs. 1 and 4). Exactly at this temperature the outer particles start to form one broad ring, as shown in Fig. 4(b), while the inner particles remain practically frozen. Only these outer particles are responsible for the formation of this plateau. As shown by Fig. 4(c), only these outer particles contribute to the mean squared radial displacement. Even more surprisingly, at the start of this plateau, the outer particles form a more symmetric configuration. In order to show this, we define the quantity $g_{6}$ $=\left[\Sigma_{i} \cos \left(6 \theta_{i}\right)\right] / 6$, where the summation is over the particles around particle 1 [see inset of Fig. 4(a) for the definition of the angles $\theta_{i}$ ]. This quantity is maximal for a triangular configuration, namely 1 , and therefore is a measure for how close the configuration is to a symmetric and triangular con-

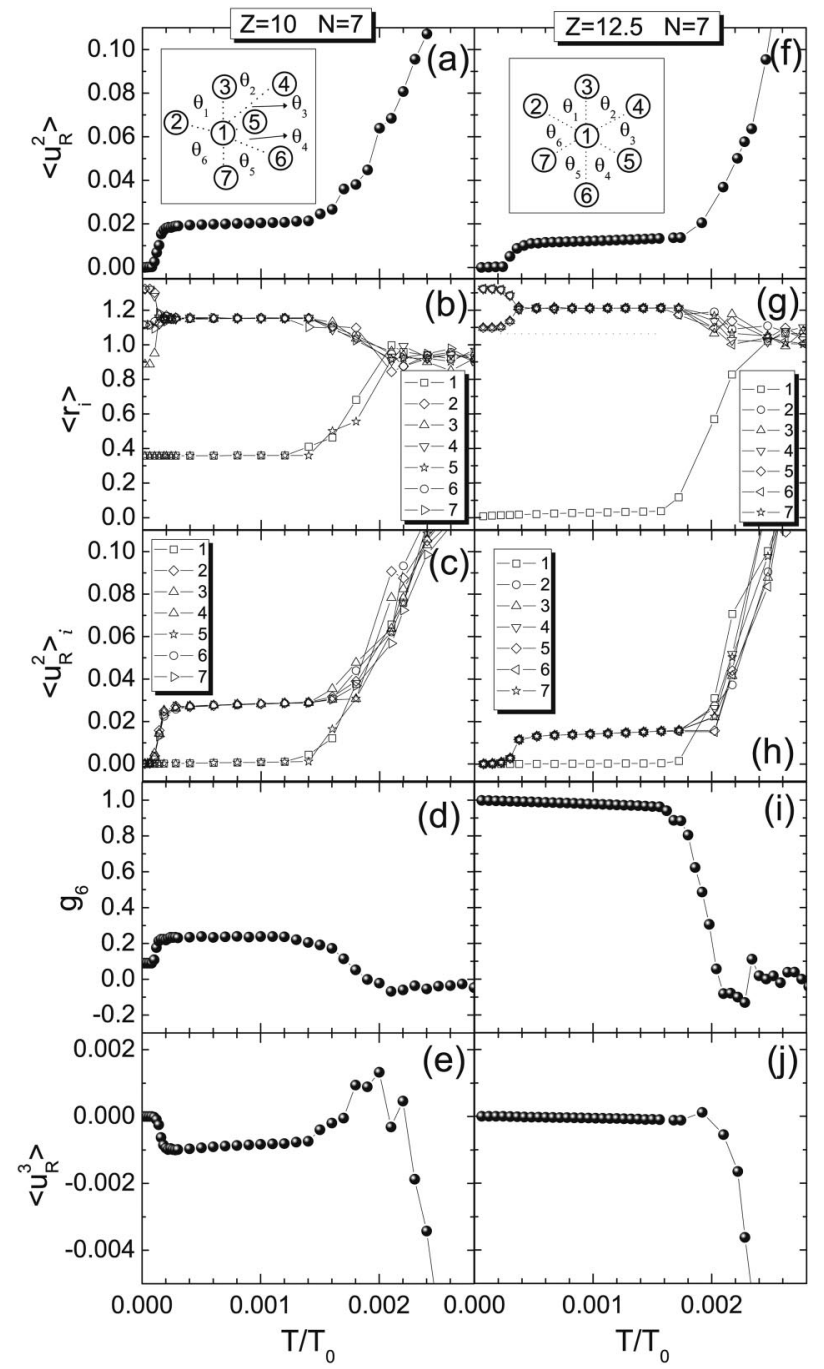

FIG. 4. Results for the asymmetric cluster $N=7, Z=10$ (left column) and the symmetric cluster $N=7, Z=12.5$ (right column): (a), (f) mean squared radial displacement; (b), (g) average position of each particle; (c), (h) mean squared radial displacement of the separate particles; (d), (i) bond angular factor; (e), (j) cubic radial displacement, as a function of temperature. The insets in (a) and (f) show the ground state configuration at $T=0$.

figuration. For the ground state of the cluster $N=7 \quad(Z=10)$, the value of $g_{6}$ is off course far from 1 . But note that it increases at the start of the plateau, indicating a more symmetric arrangement of the outer shell. These outer particles also oscillate asymmetrically around their respective equilibrium positions, as can be confirmed by the cubic radial displacement $\left\langle u_{R}^{3}\right\rangle$, defined as

$$
\left\langle u_{R}^{3}\right\rangle=\frac{1}{N} \sum_{i=1}^{N}\left\langle\left(r_{i}-\left\langle r_{i}\right\rangle\right)^{3}\right\rangle \equiv \frac{1}{N} \sum_{i=1}^{N}\left(\left\langle r_{i}^{3}\right\rangle-3\left\langle r_{i}^{2}\right\rangle\left\langle r_{i}\right\rangle+2\left\langle r_{i}\right\rangle^{3}\right) / a^{3} .
$$

If the oscillations around the equilibrium positions are symmetric, the quantity $\left\langle u_{R}^{3}\right\rangle$ should be zero, while in the opposite case, $\left\langle u_{R}^{3}\right\rangle$ is different from zero. In Fig. 4(e) the cubic 
radial deviation displacement is different from zero in the same temperature interval at which the plateau appears in the curve $\left\langle u_{R}^{3}\right\rangle$, which corresponds to the region in which the system changes its configuration.

Not only very asymmetric configurations show this unusual two-step melting process; it can also be observed in symmetric configurations. Figures $4(\mathrm{f})-4(\mathrm{j})$ show the same quantities as function of temperature as in Figs. 4(a)-4(e), but now for the configuration with $N=7$ with a larger confinement charge $Z=12.5$. This increase in confinement charge results in a symmetric ground state configuration [see the inset of Fig. 4(f)]. However, a similar plateau is observed in the mean squared radial displacement of Fig. 4(f). Note that the outer shell consists of two rings, and that they form a single broad shell at the start of the plateau [see Fig. 4(g)]. Only these outer particles contribute to the mean squared radial displacement, as shown in Fig. 4(h). A difference with the asymmetric configuration is that the symmetry of the configuration does not increase at this plateau. In fact, $g_{6}$ [Fig. 4(i)] is already close to 1 before the plateau appears. Also note that the particles oscillate symmetrically around their equilibrium position because the cubic radial displacement $\left\langle u_{R}^{3}\right\rangle$ stays zero, until the system melts completely [Fig. $4(j)]$. We found that the plateau disappears if the confinement charge is increased till $Z=14$. For $Z \geqslant 14$, the ground state is symmetric as for $Z=12.5$, but the outer particles form now a single ring. Therefore we can conclude that the two-step melting process is due to the fact that the outer particles do not form a single ring, resulting in a structural phase transition to a single ring configuration with increasing temperature.

In summary, we have studied the melting phenomenon of 2D Coulomb confined clusters of charged particles. We found the surprising result that the melting temperature is not a uniform increasing function of the strength of the binding of the cluster. It was demonstrated that the symmetry of the configuration is the dominant factor which determines the melting temperature. In the case of clusters with the same configuration, the strength of the confinement determines the melting temperature. For the studied systems the melting does not occur through jumps between stable states. Instead, a two-step melting process is observed for configurations in which the outer particles do not form an exact ring. It is a consequence of transitions to new configurations that do not exist at $T=0$.

W. P. F. and G. A. F. acknowledge support from the Brazilian National Research Councils (CNPq, CAPES), and the Ministry of Planning (FINEP). Part of this work was supported by the Flemish Science Foundation (FWO-Vl), the Belgian Science Policy, the "Onderzoeksraad van de Universiteit Antwerpen" (GOA) and the EU-RTN network on "Surface electrons."
[1] J. M. Kosterlitz and D. J. Thouless, J. Phys. C 6, 1181 (1973); B. I. Halperin and D. R. Nelson, Phys. Rev. Lett. 41, 121 (1978); A. P. Young, Phys. Rev. B 19, 1855 (1979).

[2] K. Zahn, R. Lenke, and G. Maret, Phys. Rev. Lett. 82, 2721 (1999).

[3] P. S. Branício, J. P. Rino, and N. Studart, Phys. Rev. B 64, 193413 (2001).

[4] V. M. Bedanov and F. M. Peeters, Phys. Rev. B 49, 2667 (1994).

[5] A. V. Filinov, M. Bonitz, and Yu. E. Lozovik, Phys. Rev. Lett. 86, 3851 (2001).

[6] K. J. Strandburg, Bond-Orientational Order in Condensed Matter Systems (Springer, New York, 1992).

[7] An experimental related system is described in B. A. Grsybowski, H. A. Stone, and G. M. Whitesides, Nature (London) 405, 1033 (2000).

[8] N. Metropolis, A. W. Rosenbluth, M. N. Rosenbluth, A. M.
Teller, and E. Teller, J. Chem. Phys. 21, 1087 (1953).

[9] V. A. Schweigert and F. M. Peeters, Phys. Rev. B 51, 7700 (1995).

[10] V. M. Bedanov, G. V. Gadiyak, and Yu. E. Lozovik, Phys. Lett. 109A, 289 (1985).

[11] G. A. Farias and F. M. Peeters, Solid State Commun. 100, 711 (1996).

[12] W. P. Ferreira, A. Matulis, G. A. Farias, and F. M. Peeters, Phys. Rev. E 67, 046601 (2003).

[13] W. P. Ferreira, F. M. Peeters, and G. A. Farias, Phys. Rev. E 68, 066405 (2003).

[14] H. Aref and D. L. Vainnchtein, Nature (London) 392, 769 (1998).

[15] I. V. Schweigert, V. A. Schweigert, and F. M. Peeters, Phys. Rev. Lett. 84, 4381 (2000).

[16] Yu. E. Lozovik and V. M. Fartzdinov, Solid State Commun. 54, 725 (1985). 